\title{
Spectrally resolved radiometric characterisation and calibration using a supercontinuum laser in the NIR
}

\author{
L. Bünger, R.D. Taubert, K. Anhalt \\ Physikalisch-Technische Bundesanstalt (PTB), Abbestraße 2-12, 10587 Berlin, Germany \\ Lars.Buenger@PTB.de
}

\begin{abstract}
In future, the "mise en pratique for the definition of the kelvin" [1] will allow a temperature scale to be realised and disseminated using methods of primary thermometry, such as absolute radiometry.

Here, a method is presented for the determination of the absolute spectral radiance responsivity of the measuring device, e.g. a radiation thermometer or a thermal imager. To increase the signal-to-noise ratio a supercontinuum laser system $(0.45 \mu \mathrm{m}-2.4 \mu \mathrm{m})$ is used. For radiometric applications on a high metrological level the laser system was characterised and subsequently stabilised and drift compensated to better than $0.1 \%$ per hour.

Furthermore, this laser was used for detector characterisation regarding the non-linearity and size-ofsource effect (SSE). Additionally to single detector applications, the photo-response-non-uniformity (PRNU) for focal-plane-array (FPA) detectors can be determined. Together with dedicated wavelength selecting components, the supercontinuum laser enables the determination of all these characteristics with respect to their wavelength dependencies.
\end{abstract}

Key words: absolute spectral responsivity, mise en pratique, primary thermometry, radiation thermometer, supercontinuum laser

\section{Setup - supercontinuum laser for radiometric calibration}

The redefinition of the SI unit of temperature, the kelvin, is currently in progress and will allow primary radiometry to realise the temperature scale [2]. The presented method for absolute radiometric temperature determination is based on the precise knowledge of the spectral responsivity of detector based measuring systems. These systems can be filter radiometers, radiation thermometers, as well as FPA detectors. A monochromator based setup is used for the determination of the absolute spectral radiance responsivity (fig.1).

The calibration method relies on a spectral radiance/ irradiance responsivity comparison between a well-known reference detector and the detector under test. The spectral responsivity of the reference detector is traceable to the primary PTB detector standard, the cryogenic radiometer [3]. The required homogeneous radiance field is realised by an integrating sphere $(\varnothing=50 \mathrm{~mm} ;(2))$ equipped with a calibrated, precision aperture with known area. In order to determine the absolute spectral responsivity of a detector with low uncertainty, it was necessary to increase the signal-to-noise ratios of the detectors, especially for the reference detector.

Therefore, a more powerful radiation source was required compared to the typically used halogen lamp. For this reason, a supercontinuum laser in combination with Acousto-Optical-Tunable-Filters (AOTF) was used as a quasi-monochromatic, wavelength tunable source. These lasers typically emit a continuum from $0.45 \mu \mathrm{m}$ up to $2.4 \mu \mathrm{m}$ with a total optical power of more than 5 watts and a spectral power of more than $1 \mathrm{~mW} / \mathrm{nm}$. The AOTFs allow a preselection of the desired wavelength and a monochromator enables a precise spectral selection and the traceability to the wavelength scale. By using dedicated optical lenses and apertures the laser beam matches the F\#-number of the monochromator system.

In comparison to a halogen lamp the laser system achieves a 25 to 350 times higher radiance level $\left(\lambda_{\mathrm{c}}=1.55 \mu \mathrm{m}\right)$ at the integrating sphere exit port depending on the monochromator bandpass width of $10 \mathrm{~nm}$ and $1 \mathrm{~nm}$, respectively. 


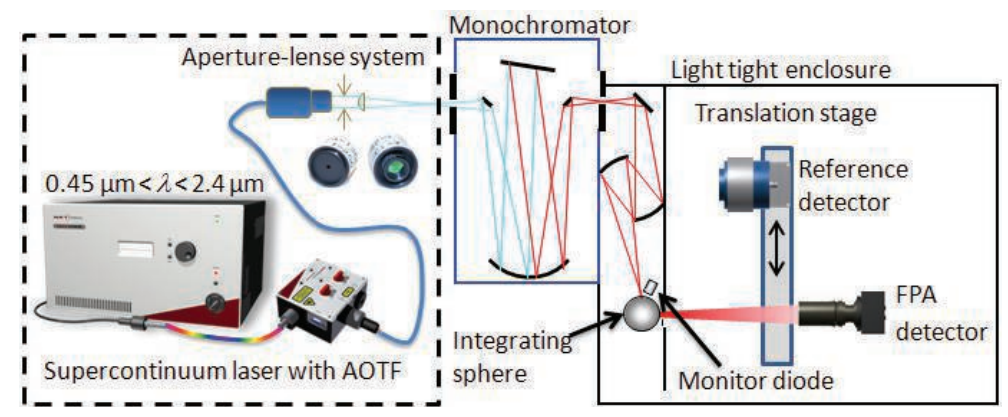

Fig. 1: Schematic of the PTB monochromator - integrating sphere based spectral comparator facility [4]. The calibration is based on a spectral radiance responsivity comparison between a well-known reference detector (e.g. trap detector) and the detector under test (e.g. FPA detector). To increase the spectral radiance at the integrating sphere exit port, a supercontinuum laser system with Acousto-Optical-Tunable-Filters (AOTF) is used.

\section{Determination of the absolute spectral radiance responsivity}

As described in the previous section, the method is based on a spectral radiance / irradiance responsivity comparison between a well-known reference detector and a detector under test. To realize the spectral radiance comparison a geometric factor $G$ (Eq.1) converts the spectral irradiance scale of the reference detector to the spectral radiance scale. The reference detector is equipped with a calibrated precision aperture with the radius $r_{2}$. The radius $r_{1}$ of the precision aperture located at the integrating sphere exit port and the distance $d$ between both apertures are also determined precisely to obtain low uncertainties for this calibration method. These parameters are also traceable to the SI. For the determination of the absolute spectral radiance responsivity an aperture with a radius $r_{1}$ of $3 \mathrm{~mm}$ was used.

$$
G=\frac{2 \pi r_{1}^{2}}{r_{1}^{2}+r_{2}^{2}+d^{2}+\sqrt{\left(r_{1}^{2}+r_{2}^{2}+d^{2}\right)^{2}-4 r_{1}^{2} r_{2}^{2}}}
$$

The absolute spectral radiance responsivity (Eq.2) of a detector under test $s_{\text {test }}^{L}$ is given by the photocurrents of the detector under test $I_{\text {test }}$ and of the reference detector $I_{\text {ref }}$ for each monochromator wavelength setting. The corresponding dark signals $\left(I_{\text {test,DS }}, I_{\text {ref,DS }}\right)$ are taken into account.

$$
s_{\text {test }}^{L}=G \frac{I_{\text {test }}-I_{\text {test,DS }}}{I_{\text {ref }}-I_{\text {ref,DS }}^{E}} s_{\text {ref }}^{E}(\lambda)
$$

The absolute spectral radiance responsivity of the detector under test must be determined over the entire spectral sensitivity range of the given sensor to avoid errors caused by out-ofband transmission of the used spectral filter.

\section{Characterisation of radiation detectors}

Single element radiation detectors are well established with respect to their technical set- up and physical response, allowing a high quality calibration and a subsequent use for measurements of radiometric quantities on the highest metrological level.

Prior to the calibration, optical radiation detectors have to be radiometrically characterised. The most significant characteristics for single element detector systems (non-imaging and imaging) are the non-linearity and the size-of-source effect (SSE). For FPA detectors additional characteristics like the photo-response-nonuniformity (PRNU) and the dark signal-nonuniformity (DSNU) have to be taken into account [5].

For the wavelength dependent characterisation with respect to SSE and PRNU a spectral tunable integrating sphere source (SUITS) was setup. This source is based on an integrating sphere irradiated by the supercontinuum laser combined with an AOTF for spectral selection (Fig. 2). The inner diameter of the sphere is $150 \mathrm{~mm}$ (6") with an output opening of $50 \mathrm{~mm}$ (2"). The AOTF provides spectrally an approximately gaussian distributed output. The bandwidth increases with increasing wavelength from $3.5 \mathrm{~nm}$ at $0.45 \mu \mathrm{m}$ to $17 \mathrm{~nm}$ at $1.7 \mu \mathrm{m}$. With a dedicated developed PID controller a radiance stability of better than $0.1 \%$ per hour was achieved.

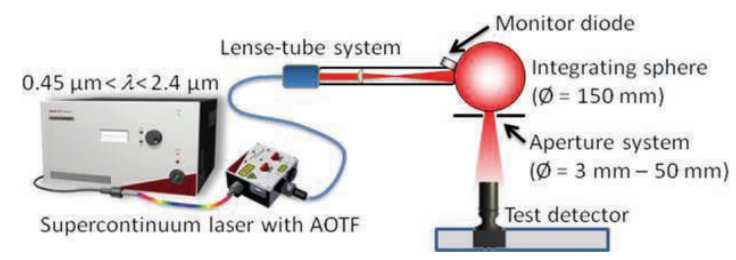

Fig. 2: Spectral tunable integrating sphere source (SUITS) based on a supercontinuum laser. The spectral selection and also the stabilisation are realised by AOTFs. The integrating sphere $(\varnothing=150 \mathrm{~mm}$, Spectralon () can be equipped with blackened apertures from $3 \mathrm{~mm}$ up to $50 \mathrm{~mm}$ or with diamond turned precision apertures for different radiometric characterisations. 


\section{Characterisation of a radiation thermometer in the NIR spectral range}

Applying the newly developed instrumentation and methodology, a radiation thermometer (RT) operating in the NIR spectral range was characterised and calibrated. This device is equipped with an InGaAs photodiode and an interference filter with a center wavelength of about $1.57 \mu \mathrm{m}$. The determined absolute spectral radiance responsivity for the bandpass region is given in Fig. 3. The responsivity in the bandpass region is 5 orders of magnitude higher than in the out-of-band region.

The non-linearity of the NIR RT is a very important characteristic due to the wide dynamic range between the spectral responsivity determination and the application for temperature measurements. The nonlinearity was determined to $4 \cdot 10^{-4}$ using the fluxdoubling method [6].

Another important characteristic of the RT is described by the SSE. The SSE is caused among others by scattered radiation incident on the detector from outside its field of view. Therefore, for the SSE determination it is necessary to use an integrating sphere based radiation source with a lager aperture than during the spectral calibration. The SSE for the radiation thermometer was determined to $3 \cdot 10^{-4}$ for apertures diameters between $3 \mathrm{~mm}$ and $75 \mathrm{~mm}$ by using the direct and also the differential method [7].

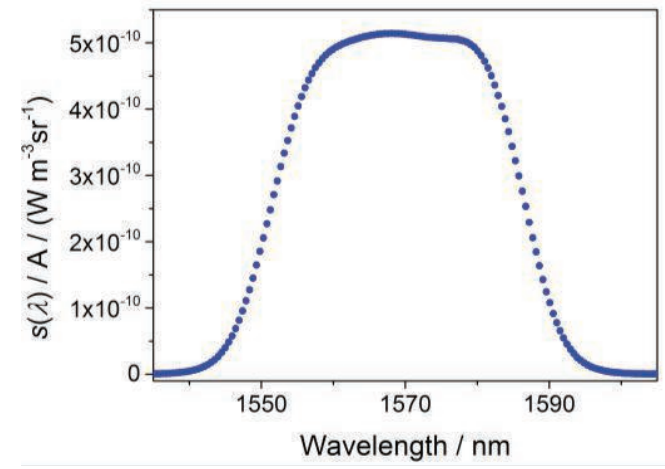

Fig. 3: Absolute spectral radiance responsivity of the NIR radiation thermometer in the bandpass region.

The preliminary combined relative standard uncertainty $(k=1)$ for the absolute spectral radiance responsivity determination was determined to $0.1 \%$. The main uncertainty components were identified to be the homogeneity of the integrating sphere, the spectral responsivity of the reference detector, and the feedback resistance value of the current-to-voltage converter.

\section{Summary}

The "mise en pratique for the definition of the kelvin" [1] allows temperature measurements based on absolute radiometry. In order to realise this primary method there is a need for absolute calibrations of radiation thermometers in the NIR spectral range. For the primary realisation of the SI unit kelvin a facility for spectral calibrations was developed. This setup is especially suitable for "radiance mode" instruments, but also can be used for "irradiance mode" instruments. All components are traceable to the SI, without the need for ITS-90 fixed-points.

For different spectral characterisations a supercontinuum laser driven spectrally tuneable integrating sphere source (SUITS) was developed. SUITS enables the spectral determination of essential radiometric characteristics like non-linearity, size-of-source effect, and photo-response-non-uniformity (for FPA detectors).

A supercontinuum laser is used to increase the radiance at the integrating sphere. The developed laser stabilisation deals with the drift compensation and decreases the noise-like fluctuation to better than $0.1 \%$ per hour. This high stability is valid for a spectral range from $0.45 \mu \mathrm{m}$ up to $1.75 \mu \mathrm{m}$ by using the laser system combined with the AOTFs.

For the validation of the new facility and the calibration methodology, a radiation thermometer operating in the near infrared spectral range was characterised and calibrated with respect to its absolute spectral radiance responsivity. The preliminary relative standard uncertainty of the absolute radiance responsivity calibration is determined to $0.1 \%$ $(k=1)$.

\section{Acknowledgement}

This work was funded by the European Association of National Metrology (EURAMET) in the framework of the European Metrology Research Programme (EMRP).

The authors thank P. Meindl for calibrating the reference detector and E. Kosubek for calibrating the precision apertures.

\section{References}

[1] Mise en pratique for the definition of the kelvin, http://www.bipm.org/utils/en/pdf/MeP_K.pdf

[2] G. Machin et al., Int J Thermophys. 31, 17791788 (2010); DOI 10.1007/s10765-010-0834-5

[3] N. Noulkow, R.D. Taubert, P.Meindl, J. Hollandt, Int J Thermophys. 30, 131-143 (2009); doi: $10.1007 / \mathrm{s} 10765-008-0458-1$ 
[4] T. Keawprasert, K. Anhalt, D.R. Taubert, J. Hartmann, Int J Thermophys. 32, 1697-1706 (2011); doi: 10.1007/s10765-011-1031-x

[5] P. Saunders, T. Ricolfi, in Proceedings of TEMPMEKO '96, 329-334 (1996)

[6] R.D. Taubert, C. Monte, K. Anhalt, L. Bünger, C. Baltruschat, in Proceedings of Tempmeko 2013

[7] P. Saunders, H. Edgar, Metrologia 46, pp. 62-74 (2009), doi: 10.1088/0026-1394/46/1/008 